\title{
Evidence-based modeling of mode-of-action for functional ingredients influencing Alzheimer's disease through neurotrophin pathway
}

\begin{abstract}
Erfan Younesi
Department of Bioinformatics, Fraunhofer Institute for Algorithms and Scientific Computing, Schloss Birlinghoven, Sankt Augustin, 53754, Germany

Corresponding author: Erfan Younesi, Department of Bioinformatics, Fraunhofer Institute for Algorithms and Scientific Computing, Schloss Birlinghoven, Sankt Augustin, 53754, Germany
\end{abstract}

Submission date: May 1, 2014; Acceptance date: August 15, 2014; Publication date: August 23,2014

\begin{abstract}
Background: Brain-derived neurotrophic factor (BDNF) is the most widely expressed member of the neurotrophin family in the human brain and is crucially involved in the development of neural circuits, modulation of synaptic plasticity, and regulation of cognitive functions, including learning and memory. Many studies have shown the association of altered BDNF levels with neurodegenerative and neuropsychiatric disorders. However, BDNF is not able to cross the blood-brain barrier and, thus, its delivery to the nervous system is a challenge. Therefore, functional diets with the ability to induce production of BDNF in the brain may offer an alternative route. The objective of this study was three-fold: first, to find out diets that are causally linked to the agonistic activity of BDNF in the neurotrophin signaling pathway; second and mainly, to investigate mode-of-action of these functional diets through systems-based mechanistic modeling in the context of Alzheimer's disease; and third, to demonstrate the proof-of-concept application of systems biology methods, that are well established in the pharmaceutical sector, to the emerging field of functional food.
\end{abstract}

Methods: In the first step, two cause-and-effect models of BDNF signaling in two states, i.e. normal state and Alzheimer's disease state, were constructed using published knowledge in scientific literature and pathway databases. A "differential model analysis" between the two states was performed by which mechanistic mode-of-action of BDNF in neurotrophin signaling pathway could be explained with a high molecular resolution in both normal and disease states. The BDNF mode-of-action model was further validated using the "biomarkerguided validation" approach. In the second step, scientific evidence on the effect of various functional diets on BDNF levels and BDNF-related biological processes or outcomes was harvested from biomedical literature using a disease-specific semantic search. This information was then added to the mechanistic model of BDNF mode-of-action and used to substantiate the mode-of-action model.

Results: The differential model analysis resulted in a mechanistic mode-of-action model for 
the effector BDNF signaling pathway through NTRK receptors (Neurotrophic tyrosine kinase receptor type 2) in neurons. The model revealed an amyloid-mediated neurotrophin switch mechanism by which the amyloid-beta protein competitively blocks BDNF-NTRK2 downstream signaling under Alzheimer's conditions, thereby "switching" the entire pathway from its normal state with neuroprotective effect to the disease state with a strong push towards neuron apoptosis. This hypothetical switch mechanism was validated by expressed biomarkers as well as empirical data obtained from experimentation of BDNF mimetics in animal models. Several functional diets were found in the literature that showed agonistic effects on the effector BDNF pathway. These effects are exerted through increased levels of BDNF and subsequently, activating the BDNF survival pathway, which leads to similar observations that have been made with BDNF mimetics in animal models.

Conclusions: To our knowledge, this is the first study to investigate mode-of-action of functional foods using systems-based modeling approaches. Moreover, such models can answer the question how functional diets can possibly act at the molecular level and interfere with the disease mechanism. Using scientific evidence supporting such models, there is a possibility to introduce new functional formulations by combining functional ingredients of these diets.

Keywords: evidence-based modeling, mode-of-action, functional ingredient, BDNF, Alzheimer's disease

\section{BACKGROUND}

Alzheimer's disease (AD) is a neurodegenerative disorder that leads to cognitive dysfunction due to the loss of neurons in the human brain. This debilitating disease imposes high economic, social and emotional burden, and presently there is no definitive diagnostic or therapeutic solutions for AD.

Neurotrophins and their receptors are among therapeutic candidates that have shown promising results for the treatment of neurological diseases. The neurotrophins are an important family of growth factors that initiate a series of molecular signals on the surface of neurons and induce the survival, development, and function of neurons through "neurotrophin signaling pathway" [1]. BDNF is the most widely expressed member of the neurotrophin family in the human brain, which binds to NTRK2 receptors and activates the neurotrophin signaling. There is accumulated evidence that BDNF exerts broad neuroprotective effects in animal models of Alzheimer's disease [2]. For example, BDNF infusion in rat and mouse has shown to reverse cognitive decline and restore memory [3]. However, neurotrophin proteins cannot be directly applied as therapeutic agents for treatment. They suffer from poor stability in serum, negligible oral bioavailability, and the pleiotropic effects; most importantly, neurotrophins are not able to cross the blood-brain barrier and penetrate the brain [4]. Therefore, to take advantage of therapeutic potential of neurotrophins, there is a need for alternative strategies.

Historically, the concept of "food as medicine" was first suggested by medieval Persian practitioners such as Avicenna and Rhazes who developed scientific guidelines on the use of natural products for treatment of diseases and health problems [5]. Functional diets enriched with natural ingredients are good candidates for circumventing pharmacological problems of 
synthetic compounds. New nutrition-based strategies should be established to intervene with the disease mechanism so that the course of disease progression is reverted to the normal condition.

Unfortunately, for the majority of food ingredients, the mechanistic mode-of-action is not known. This requires a sound understanding of disease mechanism at the molecular level and the way functional ingredients intervene with this mechanism. Systems-based integrative approaches provide a means for meeting this requirement by modeling mechanistic relations between disease pathways and health outcomes. In the present paper, we demonstrate the application of such a systems-based approach and explain the mode-of-action of several functional diets in neurotrophin pathway.

A growing body of evidence links reduced levels of neurotrophic factors to increased risk of $\mathrm{AD}$ and a number of other neurodegenerative diseases. These findings trigger the hypothesis that functional agents or ingredients that rescue the affected neurotrophin pathway (by compensating for the reduced concentrations of BDNF) may improve cognition and memory in $\mathrm{AD}$. In the following, we test this hypothesis and provide supporting evidence for dietary elements that act with a similar mode-of-action to BDNF.

\section{METHODS}

In the first step, two cause-and-effect models of BDNF signaling in two states, i.e. normal state and Alzheimer's disease state, were constructed using published knowledge in scientific literature and pathway databases. The normal BDNF pathway was reconstructed from the neurotrophin pathway in the KEGG database [6]. A part of this pathway model demonstrates downstream signaling by BDNF-NTRK2 interaction through a variety of intracellular signaling cascades, transmitting positive signals like enhanced survival and growth of neurons. This map can be accessed at www.genome.jp/kegg-bin/show_pathway?map04722. The perturbed BDNF signaling pathway under AD conditions was built by keyword search of the PubMed abstracts (Alzheimer's disease AND BDNF AND neurotrophin signaling pathway; accessed 16.07.2014). Twenty nine retrieved abstracts were manually filtered for their relevance and information content on impaired BDNF signaling under AD conditions, out of which six publications were qualified (PMIDs: 11438587, 16187222, 9106250, 21460223, 21647938, 23599427). Finally, cause-and-effect statements were extracted from these abstracts and used to draw the model. Thus, all interactions in this model are supported by scientific evidence harvested from the literature.

In the second step, a "differential model analysis" between the two states was performed by aligning the two models. The BDNF mode-of-action model was further validated using the "biomarker-guided validation" approach. In this approach, we extracted information on the potential biomarker activity (expression) of BDNF and NTRK2 from the literature, manually checked them and mapped onto the differential model.

In the third step, scientific evidence on the effect of various functional diets on BDNF levels and BDNF-related biological processes or outcomes was harvested from biomedical literature using an advanced semantic search engine, which can be freely accessed under www.scaiview.com/scaiview-academia.html. The following query was formulated: (([Human Genes/Proteins:“BDNF"]) AND [MeSH Disease:“Alzheimer Disease"]) AND [NDD:"Diet"]. Retrieved documents were manually checked and cause-and-effect information was extracted. This information was then compared to the mechanistic model of BDNF mode-ofaction and used to substantiate the mode-of-action model. 


\section{RESULTS}

The differential model analysis (BDNF-normal vs. BDNF-disease) resulted in a mechanistic mode-of-action model for the effector BDNF signaling pathway through NTRK2 receptor in neurons (Figure 1). The model revealed an amyloid-mediated neurotrophin switch mechanism by which the amyloid-beta protein competitively blocks BDNF-NTRK2 downstream signaling under Alzheimer's conditions. Simultaneously, amyloid-beta promotes neuronal death via cross-linking NGFR receptor with NTRK1 and suppressing the survivalpromoting PI3K/Akt pathway, thereby "switching" the entire pathway from its normal state with neuroprotective effect to the disease state with a strong push towards neuron apoptosis.

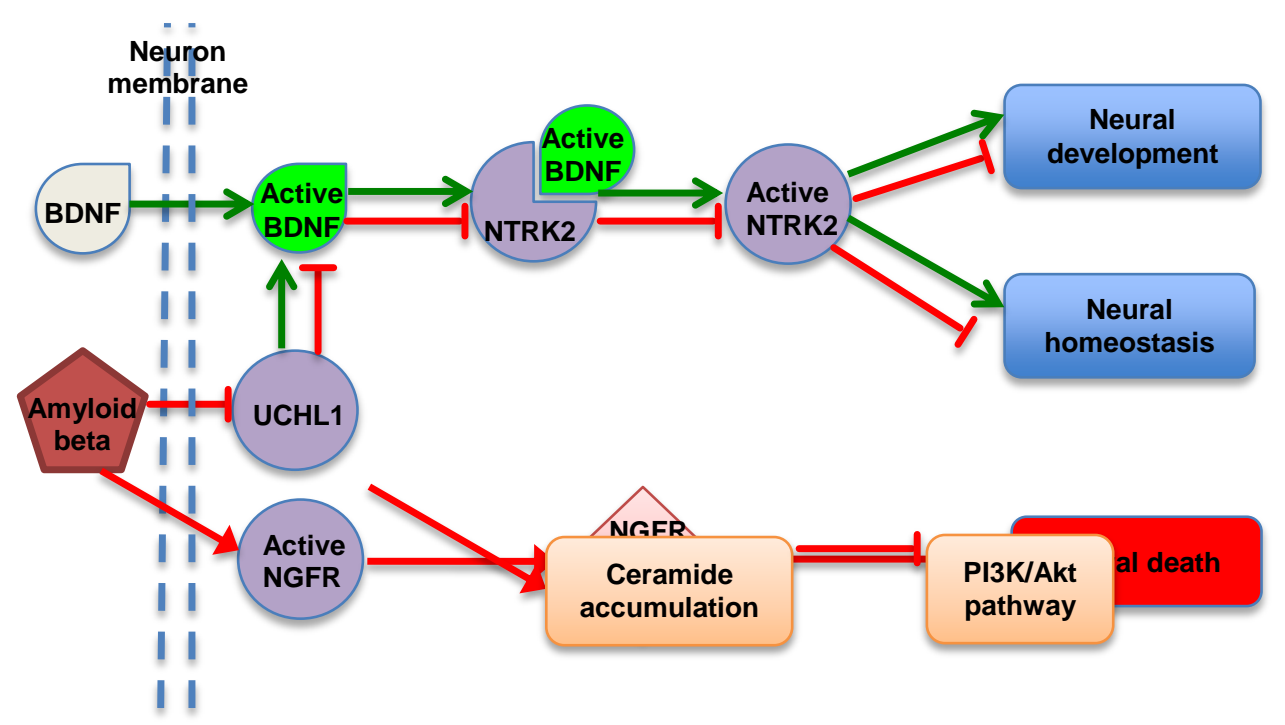

Figure 1. Schematic illustration of BDNF mode-of-action in normal and disease states. BDNF signaling in normal state has been indicated in green, whereas perturbed BDNF signaling in disease state are shown in red. This differential model uncovers the switch mechanism by amyloid-beta under AD conditions.

This hypothetical switch mechanism was validated by expressed biomarkers as well as empirical data obtained from experimentation of BDNF mimetics in animal models. Table 1 summarizes biomarker evidence for BDNF and NTRK2 receptor. As evident in this table, there are several studies that confirm BDNF and its receptor NTRK2 are downregulated in the hippocampus and cortex of AD brains.

Table 1. Biomarker evidence for BDNF and its receptor. Studies that have measured expression levels of BDNF and NTRK2 collectively confirm reduction of BDNF and NTRK2 protein levels.

\begin{tabular}{|l|l|l|l|l|l|}
\hline Protein & Expression & Brain region & Organism & $\begin{array}{l}\text { No. of } \\
\text { Evidence }\end{array}$ & PMIDs \\
\hline BDNF & $\begin{array}{l}\text { Down- } \\
\text { regulation }\end{array}$ & $\begin{array}{l}\text { Hippocampus, } \\
\text { Cortex }\end{array}$ & $\begin{array}{l}\text { Human, } \\
\text { Mouse }\end{array}$ & 7 & $\begin{array}{l}12654514,22870188, \\
20807770, \\
17990049,9387865,2044 \\
\end{array}$ \\
& & & & & 7730,1742020 \\
\hline NTRK2 & Down- & CA1 neurons, & Human, & 5 & $\begin{array}{l}22209410,20655510, \\
8931004,16539663, \\
\end{array}$ \\
& regulation & Glia, & Mouse & & \\
& & Forebrain & & & 10502038 \\
\hline
\end{tabular}


The biomarker evidence, however, can be further substantiated by the results from translational studies in which synthetic agonists of BDNF have been tested and a positive effect on the neural survival pathway has been reported. Table 2 lists studies in which BDNF agonists were reported to reverse memory impairment in mouse models of AD.

Table 2. Translational evidence supporting BDNF mode-of-action. Six studies report on the mode-of-action of BDNF agonists for improved memory and learning.

\begin{tabular}{|l|l|l|}
\hline Agent & Proof-of-Concept & Reference \\
\hline Compound J147 & $\begin{array}{l}\text { J147 inducing the BDNF protein } \\
\text { and its downstream molecules } \\
\text { reversed cognitive impairment in } \\
\text { aged AD mice. }\end{array}$ & $\begin{array}{l}\text { Prior, M., Dargusch, R., Ehren, J. L., } \\
\text { Chiruta, C., \& Schubert, D. (2013). } \\
\text { Alzheimers Res Ther, 5(3), 1-19. }\end{array}$ \\
\hline $\begin{array}{l}7,8- \\
\text { dihydroxyflavone }\end{array}$ & $\begin{array}{l}\text { 7,8-DHF a small-molecule TrkB } \\
\text { agonist, reverses memory deficits } \\
\text { and BACE1 elevation in a mouse } \\
\text { model of Alzheimer's disease. }\end{array}$ & $\begin{array}{l}\text { Devi, L., \& Ohno, M. (2012). } \\
\text { Neuropsychopharmacology, 37(2), } \\
\text { 434-444. }\end{array}$ \\
\hline $\begin{array}{l}\text { Fingolimod } \\
\text { neurotrophic factor levels and } \\
\text { ameliorates amyloid B-induced } \\
\text { memory impairment. }\end{array}$ & $\begin{array}{l}\text { Fukumoto, K., Mizoguchi, H., } \\
\text { Takeuchi, H., Horiuchi, H., } \\
\text { Suzunokuchi, J., Jin, S., ,..\& } \\
\text { brain research, 268, 88-93. }\end{array}$ \\
\hline Deoxygedunin & $\begin{array}{l}\text { Deoxygedunin imitates BDNF's } \\
\text { biological activities through } \\
\text { activating TrkB }\end{array}$ & $\begin{array}{l}\text { Jang, S. W., Liu, X., Chan, C. B., } \\
\text { France, S. A., Sayeed, I., Tang, W., .. } \\
\text { \& Ye, K. (2010). PLoS One, 5(7), } \\
\text { e11528. }\end{array}$ \\
\hline $\begin{array}{l}\text { LM22A } \\
\text { compounds }\end{array}$ & $\begin{array}{l}\text { Small molecule BDNF mimetics } \\
\text { activate TrkB signaling and prevent } \\
\text { neuronal degeneration in rodents }\end{array}$ & $\begin{array}{l}\text { Massa, S. M., Yang, T., Xie, Y., Shi, } \\
\text { J., Bilgen, M., Joyce, J. N., .. \& } \\
\text { Longo, F. M. (2010). The Journal of } \\
\text { clinical investigation, 120(5), 1774. }\end{array}$ \\
\hline
\end{tabular}

According to the above results, it can be hypothesized that any functional ingredient with BDNF boosting properties may be able to revert the neural apoptosis to the neural survival pathway.

Several functional diets were found in the literature that showed agonistic effects on the effector BDNF pathway (Table 3). These effects are exerted through increased levels of BDNF and subsequently, activating the BDNF pro-survival pathway, which leads to similar observations that have been made with BDNF mimetics in animal models. As shown in Table 3 , there are diets with both positive (agonistic) and negative (antagonistic) effects on the BDNF signaling pathway.

Table 3. List of diets/ingredients reported in the literature to agonize or antagonize the effects of BDNF

\begin{tabular}{|l|l|l|}
\hline Diet / Ingredient & Mode-of-Action & $\begin{array}{l}\text { Evidence } \\
\text { (PMID) }\end{array}$ \\
\hline Tryptophan & Increases BDNF levels in the cerebral cortex & 10192916 \\
\hline Caffeine & Preserves basal levels of BDNF & 22706686 \\
& Increased hippocampal BDNF & 23220362 \\
\hline
\end{tabular}




\begin{tabular}{|c|c|c|}
\hline Carbohydrate diet & Reduces hippocampal BDNF & 22836186 \\
\hline 2-deoxy-D-glucose & Increased expression of BDNF & 21747957 \\
\hline Cocoa polyphenols & $\begin{array}{l}\text { Triggers neuroprotection by activating BDNF survival } \\
\text { pathway }\end{array}$ & 23554028 \\
\hline High-methionine diet & Lowers levels of BDNF & 21647626 \\
\hline $\begin{array}{l}\text { High dietary } \\
\text { cholesterol }\end{array}$ & Elevated BDNF mRNA expression & 21826472 \\
\hline Alpha-lipoic acid & $\begin{array}{l}\text { Increases the expression of BDNF in the hippocampus } \\
\text { of rats }\end{array}$ & 24008266 \\
\hline High-fat diet & Reduces BDNF expression & 20176720 \\
\hline Lutein & Prevents depletion of BDNF & 22211688 \\
\hline Ginsenoside & Increased expression of BDNF & 15663889 \\
\hline
\end{tabular}

\section{DISCUSSION}

Due to lack of disease-modifying treatments and failure of recent drugs, the AD research is witnessing a focus shift from treatment to targeted prevention at both scientific and political levels [7, 8]. Healthy nutrition has been suggested as a preventive strategy in the risk reduction and management of $\mathrm{AD}[9,10]$. Epidemiological studies on relations between dietary factors and cognitive decline have shown that some nutritional ingredients such as saturated fatty acids can exacerbate cognitive decline, whereas other ingredients, including vitamins and unsaturated fatty acids, are associated with a reduced risk of dementia [11-13]. The challenge is, however, to associate epidemiological observations to underlying molecular functions for such food ingredients. Despite accumulated data and knowledge on the preventive effects of nutritional elements and dietary ingredients on $\mathrm{AD}$, their mode-of-action is not well understood. We demonstrated that systems modeling of mode-of-action for dietary ingredients could support mechanism discovery and substantiation of health outcomes in the case of neurotrophin pathway.

The neurotrophic protein BDNF and its receptor NTRK2 are involved in neuron differentiation and growth. The differential model analysis between the normal and disease signaling states in the neurotrophin pathway led to the identification of "chains of causal relationships" that led to completely new insights into putative disease mechanisms, supporting the amyloid-mediated neurotrophin switch hypothesis underlying Alzheimer's disease etiology. In the normal state, UCHL1, a de-ubiquinating enzyme that controls the BDNF-mediated retrograde transport, activates BDNF and increases the binding of BDNF to its receptor NTRK2, thereby promoting neuronal development and homeostasis. In contrast, under Alzheimer's disease conditions, amyloid-beta prevents the binding of BDNF to NTRK2 receptor, thereby blocking BDNF-NTRK2 downstream signaling. This blockade leads to repression of neuron survival, differentiation and growth, so that abnormal APP processing and amyloid-beta production has been experimentally shown to attenuate BDNFNTRK2 signaling [14]. UCHL1 activity is repressed by amyloid-beta, which in turn impairs BDNF-NTRK2-mediated downstream signaling, leading to diminished synaptic plasticity and neuronal survival [15].

Accordingly, the model was able to link the causal effect of functional diets to the BDNF mode-of-action, spanning upstream the neurotrophin pathway all the way to downstream outcomes. This was achieved by an evidence-based approach to explain mode-of-action of those dietary elements that intervene mechanistically at the molecular level with the disease pathway. 
Using such supportive scientific evidence, there is a possibility to introduce new functional formulations by combining functional ingredients of these diets. These formulations can then be used for development of functional diets that decrease the risk of AD by enhancing the neuroprotective factors responsible for the delayed onset of the disease. We believe that this approach opens up new opportunities for development of innovative functional products on the basis of sound scientific substantiation.

\section{CONCLUSIONS}

Development of innovative novel functional products requires a profound understanding of the complex relationships between the ingredient in question and its causal effect on the biological processes in the human body. Gaining insight into such a complex system is not feasible without consolidating the existing published knowledge and analytical data. It is anticipated that systems modeling strategies play an increasingly important role in development of functional food products by explaining molecular mode-of-action and substantiating expected health outcomes.

Competing interests: The author declares no competing interests.

Acknowledgments: The author wishes to acknowledge Fraunhofer Institute for Algorithms and Scientific Computing for supporting this work.

\section{REFERENCES}

1. Chao MV: Neurotrophins and their receptors: a convergence point for many signalling pathways. Nat Rev Neurosci 2003, 4:299-309.

2. Nagahara AH, Merrill DA, Coppola G, Tsukada S, Schroeder BE, Shaked GM, Wang L, Blesch A, Kim A, Conner JM, Rockenstein E, Chao MV, Koo EH, Geschwind D, Masliah E, Chiba AA, Tuszynski MH: Neuroprotective effects of brain-derived neurotrophic factor in rodent and primate models of Alzheimer's disease. Nat Med 2009, 15:331-337.

3. Li M, Dai FR, Du XP, Yang QD, Zhang X, Chen Y: Infusion of BDNF into the nucleus accumbens of aged rats improves cognition and structural synaptic plasticity through PI3K-ILK-Akt signaling. Behav Brain Res 2012, 231:146-153.

4. Longo FM, Massa SM: Small-molecule modulation of neurotrophin receptors: a strategy for the treatment of neurological disease. Nat Rev Drug Discovery 2013, 12:507-525.

5. Nikaein F, Zargaran A, Mehdizadeh A: Rhazes' concepts and manuscripts on nutrition in treatment and health care. Ancient science of life 2012, 31:160.

6. The KEGG Database [www.genome.jp/kegg/]

7. Selkoe DJ: Preventing Alzheimer's disease. Science 2012, 337:1488-1492.

8. Golubnitschaja O, Watson ID, Sandberg S, Ferrari M, Costigliola V: Position paper of the EPMA and EFLM: a global vision of the consolidated promotion of an integrative medical approach to advance health care. The EPMA journal 2013, 4:110.

9. Middleton LE, Yaffe K: Promising strategies for the prevention of dementia. Arch Neurol 2009, 66:1210-1215. 
10. Mi W, van Wijk N, Cansev M, Sijben JW, Kamphuis PJ: Nutritional approaches in the risk reduction and management of Alzheimer's disease. Nutrition 2013, 29:10801089.

11. Solfrizzi V, Panza F, Frisardi V, Seripa D, Logroscino G, Imbimbo BP, Pilotto A: Diet and Alzheimer's disease risk factors or prevention: the current evidence. Informa healthcare 2011, 11:677-708.

12. Gillette-Guyonnet S, Secher M, Vellas B: Nutrition and neurodegeneration: epidemiological evidence and challenges for future research. B J Clin Pharmacol 2013, 75:738-755.

13. Morris MC: The role of nutrition in Alzheimer's disease: epidemiological evidence. Eur J Neurol 2009, 16:1-7.

14. Ye X, Tai W, Zhang D: The early events of Alzheimer's disease pathology: from mitochondrial dysfunction to BDNF axonal transport deficits. Neurobiol Aging 2012, 33:1122-e1.

15. Poon WW, Carlos AJ, Aguilar BL, Berchtold NC, Kawano CK, Zograbyan V, Yaopruke T, Shelanski M, Cotman CW: $\beta$-Amyloid (A $\beta$ ) Oligomers Impair Brainderived Neurotrophic Factor Retrograde Trafficking by Down-regulating Ubiquitin C-terminal Hydrolase, UCH-L1. J Biol Chem 2013, 288:16937-16948. 